\title{
ON GROUPS GENERATED BY THREE-DIMENSIONAL SPECIAL UNITARY GROUPS II
}

\author{
KOK-WEE PHAN
}

(Received 19 November 1974; revised 30 October 1975)

\section{Introduction}

We shall determine in this paper groups of types $D_{n}, E_{6}, E_{7}$ and $E_{8}$ generated by $S U(3, q)$ 's, $q$ odd, $q>3$. These groups are defined in Phan (1975). [We shall refer to this paper as I]. Acquaintance with the results of $I$ is assumed. The identification of groups of type $D_{4}$ is similar to that of $S U(n, q)$. We actually construct an isomorphism from the universal group of type $D_{4}$ onto $\operatorname{Spin}^{+}(8, q)$. This direct approach does not appear to be feasible for groups of type $D_{n}$ with $n \geqq 5$. Fortunately Wong's recent result (1974) is applicable here. But his theorem requires that the characteristic of the field be odd; hence unlike the unitary case, we assume that $q$ is odd and $q>3$. Using Wong's theorem, we proceed to show by induction that groups of type $D_{n}$ are homomorphic images of $\operatorname{Spin}^{+}(2 n, q)$ or $\operatorname{Spin}^{-}(2 n, q)$ according as $n$ is even or $n$ is odd.

We then use our result on groups of type $D_{n}$ and the structure of these groups to show the existence of Steinberg's generators and relations in groups of types $E_{6}, E_{7}$ and $E_{8}$. It turns out that these are either Chevalley groups or their twisted analogues.

\section{Groups of types $D_{n}$}

Let $U$ be a vector space of dimension $m$ over a field $K$ of odd characteristic and $f$ a non degenerate symmetric bilinear form on $U$. The set of isometries forms the orthogonal group $O_{m}(K, f)=O(U)$. The subgroup of determinant 1 of the orthogonal group and the commutator subgroup $O(U)^{\prime}$ are denoted by $S O(K, f)=S O(U)$ and $\Omega(U)$ respectively. When $m$ is even and $K$ is finite, there are two non equivalent symmetric bilinear forms giving rise to non isomorphic orthogonal groups. When $K$ is finite of order $q$ and the 
index of $f$ is $m / 2$, we also denote $\Omega(K, f)$ by $\Omega^{+}(m, q)$. In the case that the index of $f$ is $m / 2-1$ and $|K|=q$ we denote $\Omega_{m}(K, f)$ by $\Omega^{-}(m, q)$. The corresponding subgroups $\operatorname{Spin}_{m}(K, f)=\operatorname{Spin}(U)$ of the Clifford group are denoted by $\operatorname{Spin}^{+}(m, q)$ and $\operatorname{Spin}^{-}(m, q)$ respectively [Dieudonné (1955)].

We shall next show that $\Omega_{m}(K, f)$ can be embedded in the special unitary group of some hermitian space depending on $m$ and $f$. Let $V$ be a non degenerate hermitian space of dimension $2 n \geqq 4$ over the finite field $F$ of $q^{2}$ elements. We shall assume throughout this paper that $q$ is odd and $q>3$. We denote the hermitian form by (, ). Let $B=\left\{v_{1}, v_{2}, \cdots, v_{2 n}\right\}$ be an orthonormal basis of $V$. Let $L_{1}^{*}\left(\operatorname{resp} . \Gamma_{1}^{*}\right), 1 \leqq i \leqq n-1$ denote the subgroup of $S U(V)$ whose restriction to the subspace $V_{i}=\left\{v_{2 i-1}, v_{2 i}, v_{2 i+1}, v_{2 i+2}\right\}$ is represented by the matrices

$$
\left(\begin{array}{rrrr}
\alpha & 0 & \beta & 0 \\
0 & \bar{\alpha} & 0 & \bar{\beta} \\
-\bar{\beta} & 0 & \bar{\alpha} & 0 \\
0 & -\beta & 0 & \alpha
\end{array}\right) \quad \text { resp. } \quad\left(\begin{array}{rrrr}
\alpha & 0 & 0 & \beta \\
0 & \bar{\alpha} & \bar{\beta} & 0 \\
0 & -\beta & \alpha & 0 \\
-\bar{\beta} & 0 & 0 & \bar{\alpha}
\end{array}\right)
$$

$\alpha, \beta \in F, \alpha \bar{\alpha}+\beta \bar{\beta}=1 \quad\left(\bar{x}=x^{q}\right)$ and $L_{i}^{*}\left(\right.$ resp. $\left.\Gamma_{i}^{*}\right)$ fixes elementwise the orthogonal complement $V_{i}^{1}$ of $V_{i}$. Let $H_{i}^{*}$ (resp. $K_{i}^{*}$ ) denote the diagonal subgroup of $L_{i}^{*}$ (resp. $\Gamma_{i}^{*}$ ). We note that $L_{i}^{*}, \Gamma_{i}^{*}$ are isomorphic to $S U(2, q)$ and $H_{i}^{*}, K_{i}^{*}$ are cyclic of order $q+1$, and generate an abelian subgroup of $S U(V)$.

Let $V_{0}$ be the subspace of $V$ consisting of vectors whose column coordinate matrix has the form ' $\left(x_{1}, \bar{x}_{1}, x_{2}, \bar{x}_{2}, \cdots, x_{n}, \bar{x}_{n}\right)$. Clearly $V_{0}$ is $n$ dimensional over $F$. But we can also regard $V_{0}$ in the usual way as an $2 n$-dimensional space over $F_{0}$, the subfield of $q$ elements in $F$. We check that the hermitian form when restricted to $V_{0}$ induces a non degenerate symmetric bilinear form over $F_{0}$ and the subgroup $G^{*}=\left\langle L_{i}^{*}, \Gamma_{i}^{*} \mid 1 \leqq i \leqq n-1\right\rangle$ is faithful on $V_{0}$. Moreover the elements of $G^{*}$ are isometries of $V_{0}$ and hence $G^{*} \subseteq \Omega\left(V_{0}\right)$ as each $L_{i}^{*}, \Gamma_{i}^{*}$ is a perfect group. We verify that $V_{i}$ contains two dimensional totally degenerate subspaces (over $F_{0}$ ) e.g. $\left\{x v_{2 i-1}+\bar{x} v_{2 i}+\right.$ $\left.\sigma x v_{2 i+1}+\overline{\sigma x} v_{2 i+2}\right\}$ where $x \in F$ and $\sigma \bar{\sigma}=-1$. It also contains an anisotropic space of dimension 2 e.g. $\left\{x v_{2 i-1}+\bar{x} v_{2 i}\right\}$. Therefore $V_{0}$ has index $n$ when $n$ is even and index $n-1$ when $n$ is odd. We collect these facts in the following

Lemma 1.1. The space $V_{0}$ with the form (, $) \mid V_{0}$ is a non singular orthogonal space of dimension $2 n$ over $F_{0}$ and index $n$ or $n-1$ according as $n$ is even or odd. The group $G^{*}=\left\langle L_{i}^{*}, \Gamma_{i}^{*} \mid 1 \leqq i \leqq n-1\right\rangle$ is a subgroup of $\Omega\left(V_{0}\right)$. 
REMARK. The space $V_{0}$ is always regarded as over $F_{0}$ unless otherwise specified.

A simple computation produces the following

LEMMA 1.2. Let $s_{1}, r_{i}$ be elements of $\Gamma_{i}^{*}, L_{i}^{*}$ respectively such that $s_{1}\left(v_{1}\right)=v_{4}, s_{1}\left(v_{4}\right)=-v_{i} ; r_{i}\left(v_{2 i-1}\right)=v_{2 i+1}, r_{i}\left(v_{2 i+1}\right)=-v_{2 i-1}$. We define inductively $s_{i+1}=r_{i} s_{i} r_{i+1} s_{i}^{-1} r_{i}^{-1}, 1 \leqq i \leqq n-2$. Then

(i) $\quad \Gamma_{i+1}^{*}=r_{i} s_{i} L_{i+1}^{*} s_{i}^{-1} r_{i}^{-1}$ and hence $G^{*}=\left\langle\Gamma_{1}^{*}, L_{i}^{*} \mid 1 \leqq i \leqq n-1\right\rangle$;

(ii) $\left\langle L_{i}^{*}, L_{i-1}^{*}\right\rangle,\left\langle L_{i}^{*}, L_{i-1}^{*}\right\rangle,\left\langle L_{i}^{*}, \Gamma_{i-1}^{*}\right\rangle$ and $\left\langle L_{i}^{*}, \Gamma_{i+1}^{*}\right\rangle$ are isomorphic to $S U(3, q)$;

(iii) Statement (ii) with $L_{i}^{*}$ replaced by $\Gamma_{i}^{*}$;

(iv) $\left[L_{i}^{*}, L_{j}^{*}\right]=\left[L_{i}^{*}, \Gamma_{j}^{*}\right]=\left[\Gamma_{i}^{*}, \Gamma_{j}^{*}\right]=\left[L_{i}^{*}, \Gamma_{i}^{*}\right]=1, j \neq i-1, i, i+1$;

(v) $\left\langle L_{i}^{*}, H_{i-1}^{*}\right\rangle,\left\langle L_{i}^{*}, H_{i+1}^{*}\right\rangle,\left\langle L_{i}^{*}, K_{i-1}^{*}\right\rangle,\left\langle L_{i}^{*}, K_{i+1}^{*}\right\rangle$ are isomorphic to $G U(2, q)$;

(vi) Statement (v) with $L_{i}^{*}$ replaced by $\Gamma_{i}^{*}$;

(vii) $H_{i}^{*} H_{j}^{*}=H_{i}^{*} \times H_{j}^{*} ; H_{i}^{*} K_{j}^{*}=H_{i}^{*} \times K_{j}^{*} ; K_{i}^{*} K_{j}^{*}=K_{i}^{*} \times K_{j}^{*}, i \neq j$.

LEMMA 1.3. Let $\tilde{L}_{i}, \tilde{\Gamma}_{i}$ be the commutator subgroup of the inverse images of $L_{i}^{*}, \Gamma_{i}^{*}$ in Spin $\left(V_{0}\right)$ respectively and $\tilde{H}_{i}, \tilde{K}_{i}$ the intersection of $\tilde{L}_{i}, \tilde{\Gamma}_{i}$ with the inverse images of $H_{i}^{*}, K_{i}^{*}$ in Spin $\left(V_{0}\right)$ respectively. Set $G=\left\langle\tilde{L}_{i}, \tilde{\Gamma}_{i}\right| 1 \leqq i \leqq$ $n-1\rangle$. Let $n_{i}, p_{i}$ be representatives of inverse images of $r_{i}, s_{i}$ in $\operatorname{Spin}\left(V_{0}\right)$ respectively. Then (i)-(vii) remain valid with $L_{i}^{*}, \Gamma_{i}^{*}, H_{i}^{*}, K_{i}^{*}, r_{i}, s_{i}$ replaced by $\tilde{L}_{i}, \tilde{\Gamma}_{i}, \tilde{H}_{i}, \tilde{K}_{i}, n_{i}, p_{i}$ respectively. Moreover $\tilde{L}_{i} \cong L_{i}^{*} \cong \Gamma_{i}^{*} \cong \tilde{\Gamma}_{i}, \tilde{H}_{i} \cong H_{i}^{*} \cong K_{i}^{*} \cong$ $\tilde{K}_{i}$ and $G /\langle z\rangle$ is isomorphic to $G^{*}$ where $z$ is the product of the involutions in $\tilde{L}_{1}$ and $\tilde{\Gamma}_{1}$.

Proof. First we note that Spin $\left(V_{0}\right)$ is a non splitting central extension of a subgroup of order 2 by $\Omega\left(V_{0}\right)$. Since both $S U(2, q)$ and $S U(3, q)$ have trivial Schur multipliers (except $S U(2,9)$, whose Schur multiplier has order 3), it follows the inverse image in Spin $\left(V_{0}\right)$ of a subgroup in $\Omega\left(V_{0}\right)$ isomorphic to $S U(2, q)$ or $S U(3, q)$ is a direct product [Griess (1972)]. The assertions are now clear.

Corollary 1.4. The groups $\tilde{G}$ and $G^{*}$ are groups of type $D_{n}$ generated by $S U(3, q)$ 's.

LEMMA 1.5. $G^{*}=\Omega\left(V_{0}\right)$ and $\tilde{G}=\operatorname{Spin}\left(V_{0}\right)$.

Proof. We shall prove the lemma by induction on $n$. The cases $n=2$ and 3 are clear by 1 . Assume then $n>3$. Let $U_{1}=\left\langle v_{i} \mid 1 \leqq i \leqq 2 n-2\right\rangle \cap V_{0}$; $U_{2}=\left\langle v_{i} \mid 3 \leqq i \leqq 2 n-2\right\rangle \cap V_{0} ; \quad U_{3}=\left\langle v_{i} \mid 3 \leqq i \leqq 2 n\right\rangle \cap V_{0}$ and $U_{0}=$ $\left\langle v_{1}, v_{2}\right\rangle \cap V_{0}$. We shall regard $\Omega\left(U_{i}\right)$ as a subgroup of $\Omega\left(V_{0}\right)$ in a natural way. 
Let $g \in \Omega\left(V_{0}\right)$. The projection of $g\left(U_{0}\right)$ into $U_{3}$ is a subspace of dimension at most two. As $U_{2}$ has index at least 2 it contains all possible symmetric bilinear spaces of dimension $\leqq 2$. By Witt's theorem, we can choose suitable elements $a \in \Omega\left(U_{3}\right)$ and $b \in \Omega\left(U_{1}\right)$ such that ag $\left(U_{0}\right) \subseteq U_{1}$ and $($ bag $)\left(U_{0}\right) \subseteq U_{0}$. Since $\left.H_{1}^{*}\left\langle r_{1} s_{1}\right\rangle\right|_{U_{t 1}} \cong O\left(U_{0}\right)$, we can assume bag $\left.\right|_{U_{o}}=$ identity. It follows that bag $\in \Omega\left(U_{3}\right)$ and therefore $g \in \Omega\left(U_{3}\right) \Omega\left(U_{1}\right) \Omega\left(U_{3}\right)$. The result now follows by induction.

Remark. It was Wong (1974) who first identified the group $G^{*}$.

Since we are assuming that $q$ is odd, we can give a weaker definition of a group of type $X$ generated by $S U(3, q)$ 's. That is the set of subgroups $L_{\mathrm{i}}$ satisfies the following

(a) $G=\left\langle L_{i} \mid i \in X\right\rangle$;

(b) $\left[L_{i}, L_{j}\right]=1$ if $\{i, j\}$ is not an edge;

(c) $\left\langle L_{i}, L_{j}\right\rangle \cong S U(3, q)$ if $\{i, j\}$ is an edge;

(d) $\left[Z\left(L_{i}\right), Z\left(L_{i}\right)\right]=1$ for all $i, j$ in $X$.

Because there is only one class of four groups in $S U(3, q)$, it follows immediately that there exists cyclic subgroup $H_{i}$ of order $q+1$ such that $H_{i} H_{j}=H_{i} \times H_{j}$ and $\left\langle L_{i}, H_{j}\right\rangle \cong\left\langle L_{j}, H_{i}\right\rangle \cong G U(2, q)$ if $\{i, j\}$ is an edge.

We shall now investigate universal group $G$ of type $D_{4}$. Clearly universal groups of types $D_{2}$ and $D_{3}$ are $S U(2, q) \times S U(2, q)$ and $S U(4, q)$ respectively by I. Let the graph of $G$ be

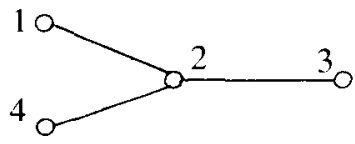

By (1.5) of I we have

$$
\left\langle L_{1}, L_{2}, L_{3}\right\rangle \cong\left\langle L_{1}, L_{2}, L_{4}\right\rangle \cong\left\langle L_{4}, L_{2}, L_{3}\right\rangle \cong S U(4, q) .
$$

Let $U$ be a non degenerate hermitian space over $F$ with orthonormal basis $\left\{u_{1}, u_{2}, u_{3}, u_{4}\right\}$. We may then regard $S U(U)$ as generated by the subgroups

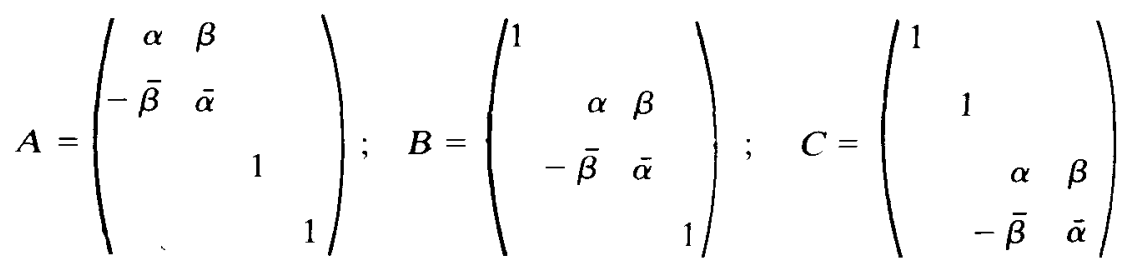

$\alpha, \beta \in F$ and $\alpha \bar{\alpha}+\beta \bar{\beta}=1$. Thus we may identify $L_{1}, L_{2}, L_{3}$ with $A, B, C$, 
respectively and $H_{1}, H_{2}, H_{3}$ with the diagonal subgroups of $A, B, C$ respectively. We have similar identification in the other two cases.

LEMMA 1.6. Let $g$ and $g^{\prime}$ be in $S U(U)$. Then one of the following holds

(i) $g \in C B A B A C$;

(ii) there exists $c \in C$ such that $g c \in A C B C B A B A$ and $c^{-1} g^{\prime} \in$ $B A B C B A B A$;

(iii) $g$ has the form

$$
\left(\begin{array}{cccc}
1 & 0 & \alpha & \sigma \alpha \\
0 & 1 & \lambda \alpha & \lambda \sigma \alpha \\
\times & \times & \times & \times \\
\times & \times & \times & \times
\end{array}\right)^{\mathrm{ab}}
$$

for a suitable $a \in A$ and $a$ diagonal element $b \in B .(\times$ denotes an unspecified entry in the matrix).

ProOF. If $g(u) \in\left\langle u_{3}, u_{4}\right\rangle$ for some $u \in\left\langle u_{3}, u_{4}\right\rangle$ of unit length, then there exist $c_{1}, c_{2}$ in $C$ such that $c_{2}\left(u_{4}\right)=u$ and $c_{1} g c_{2}\left(u_{4}\right)=u_{4}$. Then $g \in C B A B A C$ as the stabilizer of $u_{4}$ in $S U(U)$ is $\langle A, B\rangle=B A B A$. Therefore we may assume $g\left(u_{i}\right) \notin\left\langle u_{3}, u_{4}\right\rangle, i=3,4$. We now choose an element

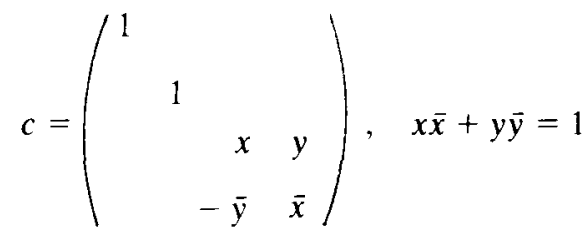

with $y \neq 0$ and $x=\zeta \bar{y}$. Let pr be the projection map into $\left\langle u_{1}, u_{2}\right\rangle$. Suppose

$$
\begin{aligned}
& \operatorname{pr} g\left(u_{3}\right)=\alpha u_{1}+\beta u_{2} \\
& \operatorname{pr} g\left(u_{4}\right)=\gamma u_{1}+\delta u_{2} .
\end{aligned}
$$

Then pr $g c\left(u_{4}\right)=y\left\{(\alpha+\gamma \bar{\zeta}) u_{1}+(\beta+\delta \bar{\zeta}) u_{2}\right\}$ which has length

$$
L=y \bar{y}\{\alpha \bar{\alpha}+\beta \bar{\beta}+(\alpha \bar{\gamma}+\beta \bar{\delta}) \zeta+(\bar{\alpha} \gamma+\bar{\beta} \delta) \bar{\zeta}+(\gamma \bar{\gamma}+\delta \bar{\delta}) \zeta \bar{\zeta}\}
$$

For (ii) to hold, we must be able to choose $\zeta$ such that it does not satisfy the equations $1+\zeta \bar{\zeta}=0 ; L=0$ and a non trivial polynomial in $\zeta$ of degree at most $q+1$ which expresses the length of the projection of $c^{-1} g^{\prime}\left(u_{4}\right)$ into $\left\langle u_{1}, u_{2}, u_{3}\right\rangle$. (See [I; 1.7] for details). Clearly such $\zeta$ exists if $L=0$ is non trivial and if $q^{2}-3(q+1)>0$ that is $q>4$. Thus it remains to consider the case when $L$ is identically zero i.e. 


$$
\alpha \bar{\alpha}+\beta \bar{\beta}=\gamma \bar{\gamma}+\delta \bar{\delta}=\alpha \bar{\gamma}+\beta \bar{\delta}=0 .
$$

We may assume none of $\alpha, \beta, \gamma, \delta$ is zero; otherwise we are back to the situation of (i). Thus $\beta=\lambda \alpha \neq 0$ where $\lambda \bar{\lambda}=-1$. Equation (2) shows that $\alpha u_{1}+\beta u_{2}$ and $\gamma u_{1}+\delta u_{2}$ are non zero isotropic vectors orthogonal to each other. Because $\left\langle u_{1}, u_{2}\right\rangle$ is not totally degenerate, it follows that $\gamma=\sigma \alpha$ and $\delta=\sigma \beta$ for some $\sigma \in \dot{F}$. If $\sigma \bar{\sigma}=-1$, then there exists $c^{\prime} \in C$ such that pr $g c^{\prime}\left(u_{4}\right)=0$ and we are again in (i). So $\sigma \bar{\sigma}=-1$. It follows then the projections of $g^{-1}\left(u_{1}\right)$ and $g^{-1}\left(u_{2}\right)$ into $\left\langle u_{1}, u_{2}\right\rangle$ are orthogonal vectors of unit length. It is now clear that (iii) follows. This completes the proof.

LEMMA 1.7. Let $G$ be a universal group of type $D_{4}$ generated by $S U(3, q)$ 's with the following graph

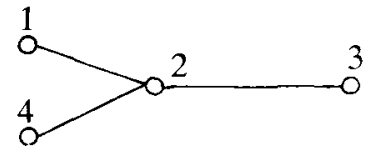

Then $G=\left(N L_{3}\right)^{3} N$ where $N=\left\langle L_{1}, L_{2}, L_{4}\right\rangle$.

Proof. We have already remarked that

$$
\left\langle L_{1}, L_{2}, L_{4}\right\rangle \cong\left\langle L_{1}, L_{2}, L_{3}\right\rangle \cong\left\langle L_{4}, L_{2}, L_{3}\right\rangle \cong S U(4, q) .
$$

Let $M=L_{2} L_{1} L_{2} L_{4} L_{2} L_{1} L_{2}$. Then each element $g$ in $G$ belongs to $N\left(L_{3} M\right)^{m} N$ for some integer $m>0$ since we have the following identities

$$
\left\langle L_{1}, L_{2}\right\rangle=L_{1} L_{2} L_{1} L_{2}=L_{2} L_{1} L_{2} L_{1}
$$

and

$$
\begin{aligned}
N & =L_{4}\left\langle L_{1}, L_{2}\right\rangle L_{4}\left\langle L_{1}, L_{2}\right\rangle \\
& =\left\langle L_{1}, L_{2}\right\rangle L_{4}\left\langle L_{1}, L_{2}\right\rangle L_{4}
\end{aligned}
$$

by (1.7) of I. We also need the identity

$$
\begin{aligned}
N & =\left\langle L_{1}, L_{2}\right\rangle\left\langle L_{2}, L_{4}\right\rangle\left\langle L_{1}, L_{2}\right\rangle \\
& =\left\langle L_{2}, L_{4}\right\rangle\left\langle L_{1}, L_{2}\right\rangle\left\langle L_{2}, L_{4}\right\rangle .
\end{aligned}
$$

Let $Y=N L_{3} M L_{3} M L_{3} N$. We want to show $Y=G$. It suffices to prove that an element

$$
x=c_{1} m_{1} c_{2} m_{2} c_{3} m_{3} c_{4}
$$

belongs to $Y$ where $c_{i} \in L_{3}, m_{i} \in M$. First we may assume that $c_{i} \notin H_{3}$, otherwise we are done. Let $m_{i}=b_{4 i-3} a_{2 i-1} b_{4 i}, d_{i} b_{4 i-1} a_{2 i} b_{4 i}$ where $a_{j} \in L_{1}$, $b_{j} \in L_{2}$ and $d_{j} \in L_{4}$. 
In the remaining proof we shall use the letters $a, b, c, d$ to denote arbitrary elements of $L_{1}, L_{2}, L_{3}, L_{4}$ respectively. Since we shall be interested in the factorization of $G$ only, we use the same letter in an equation to denote possible different elements. We use $y \equiv z$ to denote $N y N=N z N$. On many occasions, we need to introduce suitably chosen fixed elements in $L_{3}$. These will be denoted by $c^{*}, \tilde{c}, \bar{c}$ etc. We look at different forms of the element $x$.

(i) We may suppose $b_{1} a_{1} b_{2} d_{1} b_{3} a_{2} b_{4}$ satisfies either (i) or (ii) of (1.6).

Suppose not. We may identify $\left\langle L_{1}, L_{2}, L_{3}\right\rangle$ (resp. $\left.\left\langle L_{1}, L_{2}, L_{4}\right\rangle\right)$ with $S U(U)$ so that $L$ is identified with $A, L_{2}$ with $B$ and $L_{3}$ (resp. $L_{4}$ ) with $C$. After suitable changes in the $c_{i}, m_{i}$ of $c_{2} m_{2} c_{3} m_{3} c_{4}$ using (1) and (2), we may suppose

$$
z=\left(\begin{array}{cccc}
1 & 0 & \rho & \sigma \rho \\
0 & 1 & \lambda \rho & \lambda \sigma \rho \\
\times & \times & \times & \times \\
\times & \times & \times & \times
\end{array}\right)
$$

for some $\rho, \sigma, \lambda \in \dot{F}$ such that $\lambda \bar{\lambda}=\sigma \bar{\sigma}=-1$. Let

$$
c_{2}=\left(\begin{array}{rrrr}
1 & & & \\
& 1 & & \\
& & \eta & \tau \\
& & -\bar{\tau} & \bar{\eta}
\end{array}\right) \text { and } c^{*}=\left(\begin{array}{rrrr}
1 & & & \\
& 1 & & \\
& & x & y \\
& & -\bar{y} & \bar{x}
\end{array}\right)
$$

where $\eta \bar{\eta}+\tau \bar{\tau}=1=x \bar{x}+y \bar{y}$ and $\zeta y=\bar{x} \neq 0$. By (1.7) of Phan (1976), there exists suitable $\zeta$ such that

$$
\left(c^{*}\right)^{-1} b_{7} a_{4} b_{8} c_{3} b_{9} a_{5} b_{10} \text { and } c_{2} b_{5} a_{3} b_{6} c^{*}
$$

belong to $\left\langle L_{1}, L_{2}\right\rangle L_{3}\left\langle L_{1}, L_{2}\right\rangle$ provided $q^{2}-3(q+1)>0$. Suppose

$$
c_{2} b_{\varsigma} a_{3} b_{6} c^{*}=e c f .
$$

where $e, f \in\left\langle L_{1}, L_{2}\right\rangle$ and $c \in L_{3}$. Assume that

$$
\begin{aligned}
\left(b_{s} a_{3} b_{i}\right)\left(u_{3}\right) & =\alpha u_{1}+\beta u_{2}+\gamma u_{3} \\
(e)\left(u_{3}\right) & =\delta u_{1}+\varepsilon u_{2}+\chi u_{3}
\end{aligned}
$$

and 


$$
c=\left(\begin{array}{cccc}
1 & & & \\
& 1 & & \\
& & x^{\prime} & y^{\prime} \\
& & -\bar{y}^{\prime} & \bar{x}^{\prime}
\end{array}\right)
$$

We note that $y^{\prime} \neq 0$ as $c_{2} \notin H_{3}$. Thus $\delta=\left(y / y^{\prime}\right) \alpha ; \varepsilon=\left(y / y^{\prime}\right) \beta$ and $\chi=$ $\left(y / y^{\prime}\right)(\eta \gamma+\tau \zeta)$. If $z e$ satisfies (i) or (ii) of (1.6), then we are done. Otherwise we have

$$
(\delta+\rho \chi) / \sigma \rho=(\varepsilon+\lambda \rho \chi) / \sigma \lambda \rho
$$

and $(\delta+\rho \chi)\left(\overline{\delta+\rho_{\chi}}\right)+\sigma \bar{\sigma} \rho \bar{\rho}=0$. This implies that $\lambda \delta=\varepsilon$ and so $\chi \bar{\chi}=1$. Then the second equation above becomes

$$
\alpha \bar{\alpha}+\bar{\alpha}(\gamma \eta+\tau \zeta) \rho+\alpha(\overline{\gamma \eta+\tau \zeta}) \bar{\rho}=0 .
$$

If $\alpha=0$, then $\beta=0$ because $\lambda\left(y / y^{\prime}\right) \alpha=\left(y / y^{\prime}\right) \beta$. Therefore $b_{5} a_{3} b_{6} \in\left\langle L_{1}, H_{2}\right\rangle$ i.e. $b_{5} a_{3} b_{6}=\bar{a} \bar{b}$ where $\bar{a} \in L_{1}$ and $\bar{b} \in H_{2}$. So

$$
\begin{aligned}
x= & c_{1} m_{1} c_{2} \bar{a} \bar{b} d_{2} b_{7} a_{4} b_{8} c_{3} m_{3} c_{4} \\
\equiv & c_{1} m c_{2}^{\prime} b_{7} a_{4} b_{8} c_{3} m_{3} c_{4} \text { for a suitable } m \text { in } M \text { and } c_{2}^{\prime} \text { in } L_{3} \\
& \text { by (1) and (2). } \\
= & (c b a b) d(b a b c b a b c b a b) d b a b c \\
= & (c b a b \tilde{c}) d(\tilde{c})^{-1}(b a b c b a b c b a b) d b a b c \\
= & (b a b c b a b a) d(b a b c b a b a) d b a b c \quad \text { by }(1.7) \text { of } \mathrm{I} .
\end{aligned}
$$

So $x \in Y$. Thus we may suppose $\alpha \neq 0$; that is, (4) is a non trivial equation in $\zeta$ of degree at most $q$. Now if $q^{2}-4 q-3>0$, there exist a suitable $\zeta$ not satisfying (4) and so this completes the proof of (i).

(ii) We may suppose $b_{7} a_{4} b_{8} c_{3} b_{9} a_{5} b_{10}$ satisfies either (i) or (ii) of 1.6.

The proof is the same as in (i).

(iii) If $b_{7} a_{4} b_{8} c_{3} b_{9} a_{5} b_{10} \in L_{3} L_{2} L_{1} L_{2} L_{1} L_{3}$, then $x \in Y$.

We have

$$
\begin{aligned}
x & =c b a b d b a b c b a b d(b a b c b a b) d b a b c \\
& =c b a b d b a b c b a b d(c b a b a c) d b a b c \\
& =\left(c b a b c^{*}\right) d\left(c^{*-1} b a b c b a b c\right) d b a b a d c b a b c \\
& \equiv(c b a b a) d(b a b c b a b a) d b a b a d c b a b c \quad \text { by }(1.7) \text { of I } \\
& =c b a b a d b a b c(b a b a d b a b a d) c b a b c \\
& =c b a b a d b a b c(d b a b d b a b a) c b a b c \\
& =c(b a b a d b a b d) c b a b d b a b a c b a b c
\end{aligned}
$$


$=d c b a b d b a b a c b a b d$ baba $a$ babc by (1) and (2)

$\equiv c b a b d(b a b a c b a b \bar{c}) d\left(\bar{c}^{-1}\right.$ babacbabc $)$

$=c b a b d b a b c b a b a d b a b c b a b a$ by (1.7) of $I$.

So $x \in Y$

(iv) If $b_{1} a_{1} b_{2} d_{1} b_{3} a_{2} b_{4} \in L_{4} L_{2} L_{1} L_{2} L_{1} L_{4}$, then $x \in Y$.

We have

$$
\begin{aligned}
x & =c(b a b d b a b) c b a b d b a b c \text { bab d babc } \\
& =c(d b a b a d) c b a b d b a b c b a b d b a b c \\
& \equiv c b a b a c d b a b d b a b c b a b d b a b c \\
& \equiv c b a b c b a b d b a b c b a b d b a b c \quad \text { by }(1) \text { and }(2) \\
& \equiv\left(c b a b c b a b c^{*}\right) d\left(c^{*-1} b a b c b a b\right) d b a b c \\
& \equiv b a b c b a b a d b a b c b a b a d b a b c \\
& \in Y
\end{aligned}
$$

(v) If $b_{1} a_{1} b_{2} d_{1} b_{3} a_{2} b_{4} \in L_{1} L_{4} L_{2} L_{4} L_{2} L_{1} L_{2} L_{1} L_{4}$, then $x \in Y$.

We have

$$
\begin{aligned}
x & =c(b a b d b a b) c b a b d b a b c \text { babd bab } c \\
& \equiv c b d b a b a d c b a b d b a b c b a b d b a b c \\
& \equiv c b d b a b c b a b d(b a b c b a b) d b a b c \text { by (1) and (2). }
\end{aligned}
$$

If the bracketed term above belongs to $L_{3} L_{2} L_{1} L_{2} L_{1} L_{3}$, then we are done by (iii). In view of (ii), we may suppose it satisfies (ii) of (1.6). Therefore

$$
\begin{aligned}
x & \equiv c b d b a b c b a b d(a c b c b a b a) d b a b c \\
& \equiv c b d(b a b c b a b a c) d b c b a b a d b a b c \\
& \equiv c b d(c b c a b a b c b c) d b c b a b a d b a b c \text { by }(3) \\
& \equiv(c b d c b c) a b a(b c b c d b c b) a b a d b a b c \quad \text { by }(3) \\
& \equiv c b c(d b d b a b a d b d) c b c(d b d b a b a d b a b) c \\
& \equiv c b c(a b a d b d b a b a) c b c(a b a d b d b a b) c \quad \text { by }(3) \\
& \equiv c b c a b a d b d(b a b a c b c b a b) d(b a b c) \text { by (1), (2) } \\
& \equiv c b c a b a d b d(b a b a c b c b a b \bar{c}) d\left(\bar{c}^{-1} b a b c\right) \\
& \equiv c b c a b a d b d b a b c b a b a d b a b c \\
& \equiv(c b c b a b) d(b a b c b a b) d b a b c \quad \text { by }(1),(2) \\
& \equiv c b a b d b a b c b a b d b a b c \quad \text { by }(1.7) \text { of I } \\
& \in Y
\end{aligned}
$$

In view of (i), (iv), (v) and (1.6), the proof is now complete.

THEOREM 1.8. A universal group $G$ of type $D_{4}$ generated by $S U(3, q)$ 's is isomorphic to $\operatorname{Spin}\left(V_{0}\right)$ where $\operatorname{dim} V_{0}=8$. 
Proof. Let the graph of $G$ be as follows

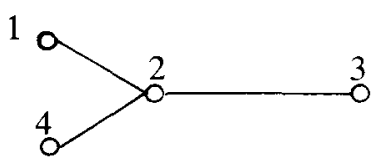

By (1.4) and (1.4) of I, we have homomorphisms

$$
G \stackrel{\phi}{\longrightarrow} \operatorname{Spin}\left(V_{0}\right) \stackrel{x}{\longrightarrow} \Omega\left(V_{0}\right)
$$

where $\operatorname{dim} V_{0}=8$ such that $\theta\left(L_{i}\right)=L_{i}^{*} i=1,2,3$ and $\theta\left(L_{4}\right)=\Gamma_{1}^{*}$ where $\theta=\chi \phi$. We observe that $z_{1} z_{4} \in \operatorname{ker} \theta$ where $z_{i} \in Z\left(L_{i}\right)^{*}$. Suppose $g \in \operatorname{ker} \theta$. We consider the following possibilities.

(i) $g=n_{1} c n_{2}$ where $n_{i} \in N=\left\langle L_{1}, L_{2}, L_{4}\right\rangle$ and $c \in L_{3}$. Then $c n \in \operatorname{ker} \theta$ where $n=n_{2} n_{1}$. Hence $\theta(c)\left(v_{8}\right)=\theta(n)^{-1}\left(v_{8}\right)=v_{\star}$. Therefore $c=1$ as $\left.\theta\right|_{L_{3}}$ is an isomorphism and the stabilizer of $v_{8}$ in $L_{3}^{*}$ is 1 . Therefore $g \in N$. On the other hand $\left.\theta\right|_{N}$ has kernel $\left\langle z_{1} z_{4}\right\rangle$. Hence $g \in\left\langle z_{1} z_{4}\right\rangle$.

(ii) $g=n_{1} c_{1} n_{2} c_{2} n_{3}$ where $n_{i} \in N$ and $c_{i} \in L_{3}$. Again we have $c_{1} n_{2} c_{2} n \in$ $\operatorname{ker} \theta$ where $n=n_{3} n_{1}$. We may suppose $c_{1}, c_{2} \notin H_{3}$, otherwise we are back to (i). Let $x=\theta\left(c_{1}\right) \theta\left(n_{2}\right) \theta\left(c_{2}\right)=\theta(n)^{-1}$. By comparing the images of $v_{8}$ (resp. $v_{7}$ ) for both expressions of $x$, we see that $\theta\left(n_{2}\right)$ fixes $\left\langle v_{6}\right\rangle$ (resp. $\left.\left\langle v_{5}\right\rangle\right)$. It follows that $n_{2} \in L_{1} L_{4} H_{2}$. Therefore $n_{2} c_{2} \in L_{3} N$ and so we are in (i).

(iii) $g=n_{1} c_{1} n_{2} c_{2} n_{3} c_{3} n_{4}$ where $n_{i} \in N, c_{i} \in L_{3}$. We may suppose none of $c_{i}$ belongs to $H_{3}$. The proof of (v) in (1.7) shows that either (i) or (ii) of (1.6) or $g$ has the form in (ii) above. Hence we may assume that $n_{2}=b_{1} d b_{2} a b_{3}$ where $a \in L_{1}, b_{i} \in L_{2}$ and $d \in L_{4}$. If one of $a, b_{1}, b_{3}, d$ belongs to $H_{1} H_{2} H_{4}$, then we may reduce the form of $g$ to case (ii) above using 1.7 of $I$ and bearing in mind the relation $L_{3} L_{2} L_{4} L_{2} L_{3} \subseteq L_{2} L_{4} L_{3} L_{2} L_{3} L_{4} L_{2} \quad((2.2)$ of $\mathrm{I}$. Let $x=\theta\left(c_{1} b_{1} d b_{2} a b_{3} c_{2}\right)=\theta\left(n_{3} c_{3} n_{4} n_{1}\right)^{-1}$. Using the second expression for $x$, we see that the projection of $x\left(v_{8}\right)$ into $\left\langle v_{7}\right\rangle$ is 0 . On the other hand, because none of $c_{1}, c_{2}, b_{1}, b_{3}, a, d$ belongs to $H_{1} H_{2} H_{3} H_{4}$, the projection of $\theta\left(c_{2}\right)\left(v_{8}\right)$ [resp. $\theta\left(b_{3} c_{2}\right)\left(v_{8}\right) ; \quad \theta\left(a b_{3} c_{2}\right)\left(v_{8}\right) ; \quad \theta\left(d b_{2} a b_{3} c_{2}\right)\left(v_{8}\right) ; \quad \theta\left(b_{1} d b_{2} a b_{3} c_{2}\right)\left(v_{8}\right) ;$ $\left.\theta\left(c_{1} b_{1} d b_{2} a b_{3} c_{2}\right)\left(v_{8}\right)\right]$ into $\left\langle v_{8}\right\rangle$ [resp. $\left.\left\langle v_{4}\right\rangle ;\left\langle v_{2}\right\rangle ;\left\langle v_{3}\right\rangle ;\left\langle v_{5}\right\rangle ;\left\langle v_{7}\right\rangle\right]$ is a non zero vector. This is a contradiction. Thus we have shown that $\operatorname{ker} \theta=\left\langle z_{1} z_{4}\right\rangle$ and $\phi$ is an isomorphism. This completes the proof.

THEOREM 1.9. Let $G$ be a universal group of type $D_{n}$ generated by $S U(3, q)^{\prime} s, n \geqq 2$. Then $G$ is isomorphic to $\operatorname{Spin}\left(V_{0}\right)$ where $\operatorname{dim} V_{0}=2 n$.

PRoOF. The result holds for $2 \leqq n \leqq 4$ as remarked earlier and by (1.7). We may suppose $n \geqq 5$. Let the graph of $G$ be as follows 


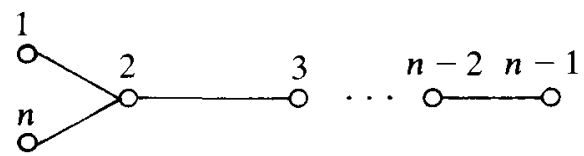

By (1.4), we have homomorphisms

$$
G \stackrel{\uplus}{\longrightarrow} \operatorname{Spin}\left(V_{0}\right) \stackrel{\phi}{\longrightarrow} \Omega\left(V_{0}\right) .
$$

Let $\theta=\phi \psi$ and $Z=\left\langle z_{1} z_{n}\right\rangle$ where $z_{i} \in Z\left(L_{i}\right)^{*}$.We shall prove by induction on $n$ that $\operatorname{ker} \theta=Z$. Thus we may suppose that $\left\langle L_{n}, L_{i} \mid 1 \leqq i \leqq n-2\right\rangle \cong$ $\operatorname{Spin}^{\epsilon}(2(n-1), q)$ where $\varepsilon=+$ if $n-1$ is even and $\varepsilon=-$ if $n-1$ is odd and $P=\left\langle L_{n}, L_{i} \mid 1 \leqq i \leqq 3\right\rangle \cong \operatorname{Spin}^{+}(8, q)$.

Let $n_{i}$ be an element of $L_{i}$ which inverts $H_{i}$. Set $\Gamma_{1}=L_{n} ; p_{1}=n_{n}$ and $K_{1}=H_{n}$. We define inductively $\Gamma_{i+1}=n_{i} p_{i} L_{i+1} p_{i}^{-1} n_{i}^{-1}$ and $K_{i+1}=$ $n_{i} p_{i} H_{i+1} p_{i}^{-1} n_{i}^{-1}$. From the isomorphism of $\left\langle L_{1}, L_{2}, \Gamma_{1}\right\rangle$ onto $S U(4, q)$ we obtain that $\left[L_{2}, \Gamma_{2}\right]=1 ; \quad n_{2} p_{2} L_{1} p_{2}^{-1} n_{2}^{-1}=\Gamma_{1} \quad$ and $\quad n_{2} p_{2} \Gamma_{1} p_{2}^{-1} n_{2}^{-1}=L_{1}$. Since $n_{1} p_{1}\left\langle L_{2}, L_{3}\right\rangle p_{1}^{-1} n_{1}^{-1}=\left\langle\Gamma_{2}, L_{3}\right\rangle$ and $n_{1} p_{1}\left\langle H_{2}, H_{3}\right\rangle p_{1}^{-1} n_{1}^{-1}=\left\langle K_{2}, H_{3}\right\rangle$, we easily verify that $M=\left\langle\Gamma_{2}, L_{i} \mid 2 \leqq i \leqq n-1\right\rangle$ is a universal group of type $D_{n-1}$ (universal because $z_{1} z_{n} \neq 1$ in $P$ and $\left.z_{2} n_{1} p_{1} z_{2} p_{1}^{-1} n_{1}^{-1}=z_{1} z_{n}\right)$. Similarly $N=\left\langle\Gamma_{4}, L_{i}\right| 4 \leqq$ $i \leqq n-1\rangle$ is a universal group of type $D_{n-3}$. We note finally from the isomorphism of $\left\langle L_{2}, L_{3}, \Gamma_{2}\right\rangle$ onto $S U(4, q), n_{3} p_{3}$ interchanges $L_{2}, \Gamma_{2}$ by conjugation. Thus $\left\langle L_{1}, L_{2}, \Gamma_{1}\right\rangle$ commutes elementwise with $\Gamma_{4}$ as $\left[L_{1}, L_{3}\right]=$ $n_{2} p_{2}\left[\Gamma_{1}, L_{3}\right] p_{2}^{-1} n_{2}^{-1}=1 ;\left[\Gamma_{1}, \Gamma_{3}\right]=1$ and $\left\langle L_{1}, L_{2}, \Gamma_{1}\right\rangle=\left\langle L_{1}, \Gamma_{2}, \Gamma_{1}\right\rangle$.

Let $V_{1}=\left\{x v_{1}+\bar{x} v_{2}\right\} ; \quad V_{2}=\left\{x v_{3}+\bar{x} v_{4}+y v_{5}+\bar{y} v_{6}\right\} ; \quad V_{3}=\left\{x v_{7}+\bar{x} v_{8}\right\}$ and $V_{4}=\left(V_{1}+V_{2}+V_{3}\right)^{\perp}, x, y \in F$. We regard $\Omega(U)$ naturally as a subgroup of $\Omega\left(V_{0}\right)$ however $U$ is a subspace of $V_{0}$. We verify that $\Omega\left(V_{1}+V_{2}\right)=$ $\theta\left(\left\langle L_{1}, \Gamma_{1}, L_{2}\right\rangle\right) ; \quad \Omega\left(V_{3}+V_{4}\right)=\theta(N) ; \quad \Omega\left(V_{1}+V_{2}+V_{3}\right)=\theta(P) \quad$ and $\Omega\left(V_{2}+V_{3}+V_{4}\right)=\theta(M)$ and $\left[\Omega\left(V_{1}+V_{2}\right), \Omega\left(V_{3}+V_{4}\right)\right]=1$. We now apply Wong's Theorem $3 \mathrm{~A}$ [Wong (1974)] and get that $G / Z \cong \Omega\left(V_{0}\right)$. Therefore $G \cong \operatorname{Spin}\left(V_{0}\right)$. The proof is now complete.

\section{Groups of types $E_{6}, E_{7}, E_{8}$}

The success in identifying groups of types $E_{6}, E_{7}$ and $E_{8}$ depends on the fact that groups of type $D_{n}$ when $n$ is even are also Chevalley groups of type $D_{n}$, and therefore they have Steinberg's generators and relations. For convenience in discussing the proof we introduce the following terminology.

Definition. Let $M_{1}, M_{2}$ be subgroups of a group $X$ with $M_{1} \cong S L(2, q)(q$ odd, $q>3$ ) such that $\left\langle M_{1}, M_{2}\right\rangle \cong S L(3, q)$ (respectively $S U(3, q)$ ) and $\left[Z\left(M_{1}\right), Z\left(M_{2}\right)\right]=1$. We say $M_{1}$ is joined to $M_{2}$ linearly (resp. unitarily) in $X$. We remark that it follows there exist cyclic subgroups $H_{i} \subseteq M_{i}$ of order $q-1$ 
(resp. $q+1$ ) such that $\left\langle H_{1}, H_{2}\right\rangle=H_{1} \times H_{2}$ and $M_{1} H_{2} \cong H_{1} M_{2} \cong G L(2, q)$ (respectively $G U(2, q)$ ).

Next we need to know the structure of a universal group $Y$ of type $D_{n}$ in some details. We introduce the following notation. Let the graph of $X$ be as follows

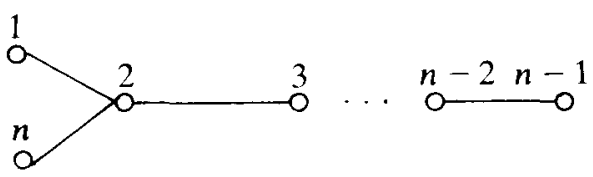

If $L_{i}$ is joined to $L_{j}$ unitarily, we can choose generators $h_{i}, h_{j}$ of $H_{i}, H_{j}$; elements $n_{i}, n_{j}$ in $L_{i}, L_{j}$ which invert $h_{i}, h_{j}$ respectively such that

$$
n_{j} h_{i} n_{j}^{-1}=h_{i} h_{j}=n_{i} h_{j} n_{i}^{-1} .
$$

Set $\Gamma_{1}=L_{n} ; p_{1}=n_{n} ; k_{1}=h_{n}$ and define inductively $\Gamma_{i+1}=x L_{i+1} x^{-1} ; p_{i+1}=$ $x n_{i+1} x^{-1} ; k_{i+1}=x h_{i+1} x^{-1}$; where $x=n_{i} p_{i}$. Let $\left\langle y_{i}\right\rangle=Z\left(L_{i}\right)$ and $\left\langle z_{i}\right\rangle=Z\left(\Gamma_{i}\right)$.

LEMMA 2.1. The following hold for the group $X$ defined above.

(i) $\left[L_{i}, L_{j}\right]=\left[L_{i}, \Gamma_{j}\right]=\left[\Gamma_{i}, \Gamma_{j}\right]=\left[L_{i}, \Gamma_{i}\right]=1$ if $j \neq i-1, i, i+1$

(ii) $L_{i}$ is joined to $\Gamma_{i-1}, L_{i-1}, \Gamma_{i+1}, L_{i+1}$ unitarily;

(iii) $\Gamma_{i}$ is joined to $\Gamma_{i-1}, L_{i-1}, \Gamma_{i+1}, L_{i+1}$ unitarily;

(iv) $p_{i} h_{i+1} p_{i}^{-1}=k_{i} k_{i+1}=n_{i+1} k_{i} n_{i+1}^{-1}$;

(v) $\quad p_{i} h_{i-1} p_{i}^{-1}=h_{i-1} k_{i}^{-1}=n_{i-1} k_{i}^{-1} n_{i-1}^{-1}$;

(vi) $k_{i+1}=h_{i} h_{i+1} k_{i}$;

(vii) $y_{i} z_{i}=y_{1} z_{1}$;

(viii) $n_{i+1} p_{i+1} L_{i} p_{i+1}^{-1} n_{i+1}^{-1}=\Gamma_{i}$.

Proof. Identifying $Y$ with Spin $\left(V_{0}\right)$ where $\operatorname{dim} V_{0}=2 n$, we verify all the assertions easily.

Definition. We call $\Gamma_{i}$ the dual of $L_{i}$ in $Y$. We note that the dual of $L_{i}$ in $Y$ is unique if $n \geqq 5$; when $n=4$, there are three subgroups $L_{3}, \Gamma_{3}, \Gamma_{1}$ which can be dual of $L_{1}$. In (2.2)-(2.5), we shall use the notation just introduced without further comment.

Lemma 2.2. Let $Y$ be the universal group of type $D_{n}$ defined above. Then $C_{Y}\left(y_{1}\right)$ contains a perfect group $C$ of index 2 which is the central product of $L_{1} \times \Gamma_{1}$ and $\left\langle L_{i}, \Gamma_{i} \mid 3 \leqq i \leqq n-1\right\rangle$ and $Z(C)=\left\langle y_{1}, z_{1}\right\rangle$.

Proof. The assertions are straightforward [Iwahori (1970)]. (Note that we are assuming $q$ odd, $q>3$.) 
Corollary 2.3. Let $x$ be an involution in $Y$ conjugate to $y_{1}$ in $Y$. Then there exists a unique subgroup $L_{x}$ in $Y$ such that $Z\left(L_{x}\right)=\langle x\rangle$.

Lemma 2.4. Let $Y$ be the universal group of type $D_{4}$, generated by $S U(3)$ 's

(i) Suppose $q \equiv 1(\bmod 4)$. Every involution $x$ in $C_{Y}\left(y_{1}\right)^{\prime}-Z\left(C_{Y}\left(y_{1}\right)\right)$ is conjugate to $n_{1} p_{1} n_{3} p_{3}$ and the unique subgroup $L_{x}$ of (2.3) is joined to $L_{1}, \Gamma_{1}, L_{3}$, $\Gamma_{3}$ linearly.

(ii) Suppose $q \equiv-1$ (mod 4). Every involution $x$ in $C_{Y}\left(y_{1}\right)-C_{Y}\left(y_{1}\right)^{\prime}$ is conjugate to $h_{2} n_{1} p_{1} n_{3} p_{3}$ and the unique subgroup $L_{x}$ of (2.3) is joined to $L_{1}, \Gamma_{1}$, $L_{3}, \Gamma_{3}$ linearly.

Proof. Since $Y \cong \operatorname{Spin}^{+}(8, q)$, we can regard $Y$ as a universal Chevalley group of type $D_{4}$ with the following Dynkin diagram.

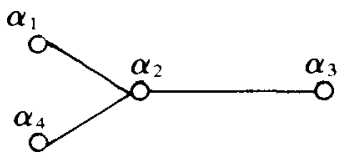

Corresponding to each root $\alpha$, we have the one parameter unipotent subgroup $\quad U_{\alpha}=\left\{x_{\alpha}(t) \mid t \in F_{0}\right\} ; \quad n_{\alpha}=x_{\alpha}(1) x_{-\alpha}(-1) x_{\alpha}(1) ; \quad h_{\alpha}(t)=$ $x_{\alpha}(t) x_{-\alpha}\left(-t^{-1}\right) x_{\alpha}(t) n_{\alpha}^{-1}$ and $X_{c \alpha}=\left\langle U_{\alpha}, U_{-\alpha}\right\rangle$. Here we can assume $y_{1}=$ $h_{\alpha_{1}}(-1)$. Then $C_{Y}\left(y_{1}\right)=C\left\langle h_{\alpha_{2}}(\lambda)\right\rangle$ where $C=X_{\alpha_{0}} X_{\alpha_{1}} X_{\alpha_{3}} X_{\alpha_{4}},\langle\lambda\rangle=\dot{F}$ and $\alpha_{0}$ the longest root. We have $h_{\alpha_{2}}(\lambda)^{2} \in C$ and $\left\langle X_{\alpha_{1}}, h_{\alpha_{2}}(\lambda)\right\rangle \cong G L(2, q), i=$ $0,1,3,4 ;\left[X_{\alpha_{i}}, X_{\alpha_{j}}\right]=1 i \neq 2 \neq j$ and $i \neq j$.

Suppose $q \equiv 1(\bmod 4)$. Then $h_{\alpha_{2}}(-1) \in C$ and $h_{\alpha_{2}}(-1) \notin Z(C)=$ $\left\langle h_{\alpha_{1}}(-1), h_{\alpha_{3}}(-1), h_{\alpha_{4}}(-1)\right\rangle$. If $x$ is an involution in $C-Z(C)$, it necessarily must have the form $x_{0} x_{1} x_{3} x_{4}$ where $x_{i} \in X_{\alpha_{i}}$ and $0\left(x_{i}\right)=4$. Since $S L(2, q)$ contains just one class of elements of order 4 , all involutions in $C-Z(C)$ are conjugate to $h_{\alpha_{2}}(-1)$. The assertions of (i) are now clear.

Next suppose $q \equiv-1(\bmod 4)$. Let $x$ be an involution in $C_{Y}\left(y_{1}\right)-$ $C_{Y}\left(y_{1}\right)^{\prime}$. Then $x$ has the form $x=h_{\alpha_{2}}(-1) y z$ when $y \in X_{\alpha_{1}}$ and $z \in X_{\alpha_{0}} X_{\alpha_{3}} X_{\alpha_{4}}$. As $X_{\alpha_{1}}\left\langle h_{\alpha}(\lambda)\right\rangle \cong G L(2, q), h_{\alpha_{2}}(-1) y$ is an involution conjugate in $Y_{\alpha_{1}}$ to $h_{\alpha_{2}}(-1)$, we may assume $y=1$. A similar argument proves that $h_{\alpha_{2}}(-1) y z$ is conjugate to $h_{\alpha_{2}}(-1)$ in $C_{Y}\left(y_{1}\right)$. This completes the proof.

LEMMA. Let $Y$ be the universal group of type $D_{6}$ generated by $S U(3)$ 's

(i) There are two classes of non central involutions with representatives $y_{1}$ and $y_{3} y_{5}$ and $C_{Y}\left(y_{1}\right)=C_{Y}\left(y_{3} y_{5}\right)$;

(ii) Suppose $x, y$ are commuting involutions conjugate to $y_{1}$ in $Y$ such that $x y$ is not conjugate to $y_{1}$ in $Y$. Let $L_{x}$ and $L_{y}$ be the unique subgroups $L_{x}$ and $L_{y}$ of (2.3) with $Z\left(L_{x}\right)=\langle x\rangle, Z\left(L_{y}\right)=\langle y\rangle$. Then $\left[L_{x}, L_{y}\right]=1$.

Proof. (i) The details can be easily computed [Iwahori (1970)]. 
To prove (ii), we introduce the usual Chevalley notation as in (2.4) since $Y \cong \operatorname{Spin}^{+}(12, q)$. We can assume $y_{1}=h_{\alpha_{1}}(-1)$ and also $x=y_{1}$. Then $L_{x}=$ $L_{1}=X_{\alpha_{1}}$. If $y \in C_{Y}\left(y_{1}\right)^{\prime}$, then $y=x_{1} x_{2}$ where $x_{1} \in L_{1}$ and $x_{2} \in \Gamma_{1} \times\left\langle L_{i}, \Gamma_{i}\right| 3 \leqq$ $i \leqq 5$ ) by (2.2). Suppose first $0\left(x_{1}\right)=0\left(x_{2}\right)=4$. From the structure of $S U(2, q)$, we get $x y=y_{1} x_{1} x_{2} \widetilde{Y} \quad x_{1} x_{2}=y$, a contradiction to our hypothesis. So we may suppose $0\left(x_{1}\right) \leqq 2$ i.e. $x_{1}=1$ or $x_{1}=y_{1}$. We compute that the classes of involutions in $\Gamma_{1} \times\left\langle L_{i}, \Gamma_{i} \mid 3 \leqq i \leqq 5\right\rangle$ have representatives $z_{1}, z_{1} y_{3} y_{5}, z_{1} y_{3} z_{5}$, $z_{1} y_{3}, y_{3} y_{5}, y_{3} z_{5}, y_{5} z_{5}, y_{3}$. Of these only those with representatives $z_{1}, y_{3}$ satisfy our requirement. In these cases we have $L_{y}=\Gamma_{1}$ or $L_{3}$ and so $\left[L_{x}, L_{y}\right]=1$.

Now suppose $y \in C_{Y}\left(y_{1}\right)-C_{Y}\left(y_{1}\right)^{\prime}$. Suppose $q \equiv 1(\bmod 4)$. Then $y$.must have the form $y=y_{2} x_{1} x_{2}$ where $x_{1} \in L_{1}$ and $x_{2} \in \Gamma_{1} \times\left\langle L_{i}, \Gamma_{1} \mid 3 \leqq i \leqq 5\right\rangle$. From the fact that $\left\langle L_{i}, h_{2}\right\rangle \cong G U(2, q)$, we find that $y_{2} x_{1}$ is an involution conjugate in $L_{1}$ to $y_{2}$. Therefore we may assume $x_{1}=1$. Then $x y=y_{1}\left(y_{2} x_{2}\right)=n_{1}\left(y_{2} x_{2}\right) n_{1}^{-1}$ $\widetilde{\gamma} y$ in contradiction to our assumption. The case $q \equiv-1(\bmod 4)$ is proved similarly regarding $Y$ as a Chevalley group and the fact $\left\langle L_{1}, h_{\alpha_{2}}(\lambda)\right\rangle \cong$ $G L(2, q)$ where $\langle\lambda\rangle=\dot{F}$. This completes the proof.

In the proofs of (2.6)-(2.8) we shall encounter certain subgroups which are homomorphic images $\bar{Y}$ of $Y=S U(m, q)$ for some integer $m>0$. For convenience, we introduce the following uniform notation for elements and subsets of $\bar{Y}$. Let $U$ be a non degenerate hermitian space of dimension $m$ on which $Y$ acts naturally. We choose an orthonormal basis $\left\{u_{1}, u_{3}, u_{4}, \cdots, u_{m+1}\right\}$. Let $L_{i j}^{*}$ be the subgroup of $Y$ which leaves $\left\langle u_{i}, u_{j}\right\rangle^{\perp}$ pointwise fixed. If $i<j$, let $h_{i j}^{*}, n_{i j}^{*}$ be the elements of $L_{i j}^{*}$ such that $h_{i j}^{*}\left(u_{i}\right)=\sigma u_{i}, h_{i,}^{*}\left(u_{j}\right)=$ $\left(\sigma^{-1}\right) u_{j}, n_{i,}^{*}\left(u_{i}\right)=u_{j}$ and $n_{i, j}^{*}\left(u_{i}\right)=-u_{i}$ where $\langle\sigma\rangle$ is the subgroup of order $q+1$ in $\dot{F}$. The images of $L_{i j}^{*}, h_{i j}^{*}, n_{i j}^{*}$ in $\bar{Y}^{\prime}$ will be denoted by $L_{i j}, h_{i j}, n_{i j}$ respectively.

Suppose that $G$ is a group of certain type generated by $S U(3, q)$ 's and $L_{i}$, $L_{j}$ are subgroups such that $L_{i}$ is joined to $L_{j}$ unitarily. We can choose generators $h_{i}, h_{j}$ of $H_{i}, H_{i}, n_{i}, n_{j}$ of $L_{i}, L_{j}$ respectively such that $n_{i}, n_{j}$ inverts $h_{i}$, $h_{j}$ and

$$
n_{i} h_{j} n_{i}^{-1}=h_{i} h_{j}=n_{j} h_{i} n_{j}^{-1} .
$$

In particular, if $G$ has a subgroup of type $A_{m-1}$ isomorphic to $\bar{Y}$ and with subgraph

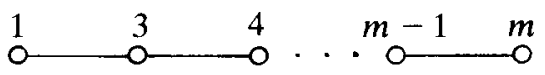

We may then identify $h_{13}, h_{i, i+1} ; n_{13}, n_{i, i+1} ; L_{i, i+1}$ with $h_{1}, h_{i} ; n_{1}, n_{i} ; L_{1}, L_{i}$ respectively. 
THEOREM 2.6. Let $G$ be a universal group of type $E_{6}$ generated by $S U(3, q)$ 's. Then $G$ is isomorphic to the twisted analogue of the universal Chevalley group of type $E_{6}$ over $F_{0}$.

Proof. Let the graph of $G$ be as follows

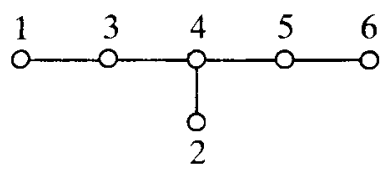

In view of (1.4) and (2.3) of I, the subgroup generated by $L_{i}, i=1,3,4,5,6$ is isomorphic to $S U(6, q)$ and so we can use the notation just introduced. Let $\theta=n_{14} n_{57}$. Then $\left[\theta, L_{2}\right]=1$ as $n_{14} \in\left\langle L_{1}, L_{3}\right\rangle$ and $n_{57} \in\left\langle L_{5}, L_{6}\right\rangle$. The element $\theta$ interchanges the elements of the sets $\left\{L_{1}, L_{3}\right\} ;\left\{L_{5}, L_{6}\right\}$ and $\left\{L_{4}, L_{17}\right\}$ by conjugation. We compute that $\theta n_{45} \theta^{-1}=n_{17}$ and $L_{17}$ is joined to $L_{2}$ unitarily as $L_{4}$ is. Since $L_{4}, L_{5}, L_{6}$ commute elementwise with $L_{17}$, it follows $N=$ $\left\langle L_{17}, L_{i} \mid 2 \leqq i \leqq 5\right\rangle \cong \operatorname{Spin}^{-}(10, q)$ as $\left(h_{3} h_{5}\right)^{q+1 / 2} \neq 1$ by (1.9). So $N \cong \operatorname{Spin}\left(V_{0}\right)$ where $V_{0}$ is the symmetric bilinear space introduced in $\S 1$. Set $V_{\mathrm{i}}=$ $\left\{x v_{2 i-1}+\bar{x} v_{2 i}+y v_{2 i+1}+\bar{y} v_{2 i+2}\right\}, \quad x, y \in F, \quad i=1,2,3,4$. We may assume $L_{3} \times L_{5}=\operatorname{Spin}\left(V_{1}\right) ; L_{4} \subseteq \operatorname{Spin}\left(V_{2}\right) ; L_{2} \subseteq \operatorname{Spin}\left(V_{3}\right)$ and $L_{17} \subseteq \operatorname{Spin}\left(V_{4}\right)$. Since $n_{34} n_{56} L_{4} n_{56}^{-1} n_{34}^{-1}=L_{36}, L_{36}$ is the dual of $L_{4}$ in $N$. So the subgroup $N_{0}=$ $\left\langle L_{17}, L_{2}, L_{4}, L_{36}\right) \cong \operatorname{Spin}^{+}(8, q)$. Let $L_{0}$ be the dual of $L_{17}$ in $N$. By (2.1), $\left[L_{0}, L_{i}\right]=1 \quad i=3,4,5$. Since $\theta N_{0} \theta^{-1}=N_{0}$, it follows $\theta L_{0} \theta^{-1}=L_{0}$ by (2.2). Therefore $\left[L_{0}, L_{1}\right]=1=\left[L_{0}, L_{6}\right]$. In particular $\left[\theta, L_{0}\right]=1$.

Let $z=n_{45} n_{17} n_{36} n_{0}$ where $n_{0}=n_{2} n_{45} n_{36} n_{2} n_{36}^{-1} n_{45}^{-1}$ when $q \equiv 1(\bmod 4)$ and $z=h_{2} n_{45} n_{17} n_{36} n_{0}$ when $q \equiv-1(\bmod 4)$. By $(2.4), z$ is an involution and there exists unique subgroup $\Gamma_{2}$ with $Z\left(\Gamma_{2}\right)=\langle z\rangle$ and $\Gamma_{2}$ is joined to $L_{17}, L_{0}, L_{36}, L_{4}$ linearly. Moreover $N_{0}=\left\langle L_{17}, L_{45}, L_{36}, L_{0}, \Gamma_{2}\right\rangle$. Furthermore $L_{45} \times L_{36}=$ $\operatorname{Spin}\left(V_{2}\right) ; L_{17} \times L_{0}=\operatorname{Spin}\left(V_{+}\right)$. Since $\left\langle\Gamma_{2}, L_{17}\right\rangle$ and $\left\langle\Gamma_{2}, L_{45}\right\rangle$ are isomorphic to $S L(3, q)$, it follows that there exist hyperbolic planes $P_{1}, P_{2}$ in $V_{2}, V_{4}$ respectively such that $\Gamma_{2} \subseteq \operatorname{Spin}\left(P_{1}+P_{2}\right)$. Let $P_{3}$ be the orthogonal complement (a hyperbolic plane) of $P_{2}$ in $V_{4}$. Thus $Q=\left(P_{1}+P_{2}+P_{3}\right)^{1}$ is a symmetric bilinear space of dimension 4 and index 1 . We note that $Q \cap V_{2}$ is a hyperbolic plane in $V_{2}$ orthogonal to $P_{1}$. Let $S_{2}=\operatorname{Spin}(Q)$. Then $S_{2} \cong$ $S L\left(2, q^{2}\right)$ [Dieudonné (1955)]; $\left\langle S_{2}, L_{4}\right\rangle=\left\langle L_{i} \mid 3 \leqq i \leqq 5\right\rangle$ and $\left[S_{2}, \Gamma_{2}\right]=1$. We note that $\left\langle S_{2}, L_{4}\right\rangle$ as a subgroup of $\operatorname{Spin}\left(V_{0}\right)$ is $\operatorname{Spin}\left(V_{1}+\left\langle x v_{5}+\bar{x} v_{6}\right\rangle\right)$. On the other hand, $\left\langle S_{2}, L_{4}\right\rangle$ regarded as a subgroup of $M$ acts on the hermitian space $\left\{u_{3}, u_{4}, u_{5}, u_{6}\right\}$. The isomorphism between $\operatorname{Spin}\left(V_{1}+\left\langle x v_{5}+\bar{x} v_{6}\right\rangle\right)$ and $S U(4, q)$ maps $Q$ to a totally degenerate subspace $U_{0}$ of dimension 2 [Dieudonné (1955)] in $\left\{\boldsymbol{u}_{3}, u_{4}, \boldsymbol{u}_{5}, u_{6}\right\}$ with $U_{0} \cap\left\langle u_{4}, \boldsymbol{u}_{5}\right\rangle=\left\langle\boldsymbol{w}_{4}\right\rangle \neq 0$ and $U_{0} \cap\left\langle\boldsymbol{u}_{3}, \boldsymbol{u}_{6}\right\rangle=$ $\left\langle w_{3}\right\rangle \neq 0$ and $U_{0}=\left\langle w_{3}, w_{4}\right\rangle$. Let $U_{1}=\theta\left\langle w_{3}, w_{4}\right\rangle=\left\langle w_{3}, \theta\left(w_{4}\right)\right\rangle$ since $\theta$ fixes 
elementwise $\left\langle\boldsymbol{u}_{3}, \boldsymbol{u}_{6}\right\rangle$. It follows $\left\langle U_{0}, U_{1}\right\rangle=\left\langle w_{3}, w_{4}, \theta\left(w_{4}\right)\right\rangle$ is a 3-dimensional totally degenerate space as $\theta\left(w_{4}\right) \in\left\langle u_{1}, u_{7}\right\rangle$. Set $S_{1}=\theta S_{2} \theta^{-1}$. Clearly $\left\langle S_{1}, S_{2}\right\rangle \cong$ $S L\left(3, q^{2}\right)$ and $\left\langle S_{1}, L_{36}\right\rangle=\left\langle L_{1}, L_{36}, L_{6}\right\rangle$ and $\left\langle S_{1}, S_{2}, L_{4}\right\rangle=M$.

We have shown $\left[\theta ; L_{2}\right]=1=\left[\theta, L_{0}\right]$ and $\theta n_{4} \theta^{-1}=n_{17}$. So $\theta z \theta^{-1}=z$ and because $\theta N_{0} \theta^{-1}=N_{0}$, therefore $\theta \Gamma_{2} \theta^{-1}=\Gamma_{2}$ by (2.3). It follows $\left[S_{1}, \Gamma_{2}\right]=1$.

We now look at the following chain of subgroups $\Gamma_{2}, L_{4}, S_{2}, S_{1}$. First they generate $G$ since $\left\langle S_{1}, S_{2}, L_{4}\right\rangle=M$ and $L_{2} \subseteq\left\langle L_{4}, \Gamma_{2}, L_{36}, L_{17}\right\rangle$. We also have the following relations $\left\langle\Gamma_{2}, L_{4}\right\rangle \cong S L(3, q) ; \quad\left[\Gamma_{2}, S_{2}\right]=1=\left[\Gamma_{2}, S_{1}\right] ; \quad\left\langle L_{4}, S_{2}\right\rangle \cong$ $S U(4, q) ;\left[L_{4}, S_{1}\right]=1\left(\right.$ as $\left\langle L_{1}, L_{36}, L_{6}\right\rangle$ centralizes $\left.L_{4}\right) ;\left\langle S_{2}, S_{1}\right\rangle \cong S L\left(3, q^{2}\right)$. It is now easy to see that the conditions of Curtis' Theorem 1.4 [Curtis (1965)] are satisfied. So $G \cong{ }^{2} E_{6}\left(q^{2}\right)$, the group of fixed points in the universal Chevalley group of type $E_{6}$ over $F$ of a 'twisting' automorphism.

Theorem 2.7. Let $G$ be a universal group of type $E_{7}$ generated by $S U(3, q)$ 's. Then $G$ is isomorphic to the universal Chevalley group of type $E_{7}$ over $F_{0}$.

Proof. Let the graph of $G$ be as follows

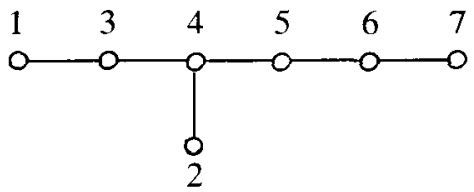

The subgroup $P=\left\langle L_{1}, L_{i} \mid 3 \leqq i \leqq 7\right\rangle$ is isomorphic to $S U(7, q)$ and $R=$ $\left\langle L_{i} \mid 1 \leqq i \leqq 6\right\rangle \cong{ }^{2} E_{6}\left(q^{2}\right)$. In the proof of (2.6), we have found the subgroup $L_{0}$ is joined to $L_{2}$ unitarily and commutes elementwise with $L_{1}, L_{i} 3 \leqq i \leqq 6$. The subgroup $N_{0}=\left\langle L_{36}, L_{45}, L_{2}, L_{0}, L_{17}\right\rangle$ is universal of type $D_{4}$. We also found the element $n_{2} n_{45} n_{36} n_{2} n_{36}^{-1} n_{45}^{-1}$ which interchanges $L_{0}$ and $L_{17}$ by conjugation (see 2.1)). Because $L_{17}$ is joined to $L_{7}=L_{78}$ unitarily, $L_{0}$ is joined to $L_{78}$ unitarily. The subgroup $S=\left\langle L_{1}, L_{0}, L_{i} \mid 3 \leqq i \leqq 7\right\rangle$ is a group generated by $S U(3, q)$ 's of type $A_{7}$ and so by (2.3) of $I S$ is a homomorphic image of $S U(8, q)$. We now use the notation introduced just prior to (2.6) and so $L_{0}=L_{89}$. As $P$ is a subgroup of $S$, the previous notation for subgroups and elements of $P$ in (2.6) is consistent with the present one.

Let $\psi=n_{17} n_{36} n_{45} n_{89}$. We compute that $\psi$ interchanges the elements of the sets $\left\{L_{1}, L_{6}\right\} ;\left\{L_{3}, L_{5}\right\},\left\{L_{19}, L_{7}\right\}$ and fixes $L_{4}, L_{89}$ by conjugation. As $\psi \in N_{0}$, we compute that $\psi$ fixes $L_{2}$ by conjugation (see also (2.1) (viii)). Therefore $\left[L_{19}, L_{2}\right]=1$ and so $L_{19}$ commutes elementwise with $L_{i}, 2 \leqq i \leqq 5$ and is joined to $L_{1}$ and $L_{89}=L_{0}$ unitarily. Thus $M_{1}=\left\langle L_{19}, L_{1}, L_{i} \mid 2 \leqq i \leqq 5\right\rangle \cong \operatorname{Spin}^{+}(12, q)$. Similarly $M_{2}=\left\langle L_{i} \mid 2 \leqq i \leqq 7\right\rangle$ and $M_{3}=\left\langle L_{78}, L_{89}, L_{i} \mid 2 \leqq i \leqq 5\right\rangle$ are isomorphic to $\operatorname{Spin}^{+}(12, q)$. 
Let $\Gamma$ be the dual of $L_{2}$ in $M_{3}$. By $(2.1),\left[L_{i}, \Gamma\right]=1 i=2,3,5,7$ and $\Gamma$ is joined to $L_{4}$ and $L_{89}$ unitarily. But $\Gamma$ is the dual of $L_{3}$ and $L_{5}$ in $M_{1}, M_{2}$ respectively. Hence $\left[L_{14}, \Gamma\right]=1$ and $\Gamma$ is joined to $L_{1}$ and $L_{6}$ unitarily. Let $\phi=n_{13} n_{49} n_{67} n_{58}$. We compute that $\phi$ interchanges the elements of the sets $\left\{L_{3}, L_{19}\right\} ;\left\{L_{4}, L_{8}\right\} ;\left\{L_{5}, L_{7}\right\}$ by conjugation. Let $t$ be the involution in $L_{2}$. Set $N_{1}=\left\langle L_{i} \mid 2 \leqq i \leqq 5\right\rangle$ and $N_{2}=\left\langle L_{2}, L_{89}, L_{19}, L_{7}\right\rangle$. These groups are isomorphic to $\operatorname{Spin}^{+}(8, q)$ and $\phi N_{1} \phi^{-1}=N_{2}$. As $C_{N_{1}}(t)^{\prime}=\Gamma L_{2} L_{3} L_{s}$ and $C_{v_{2}}(t)=\Gamma L_{2} L_{19} L_{7}$ it follows $\phi \Gamma \phi^{-1}=\Gamma$. Let $L=n_{13} n_{49} \Gamma n_{49}^{-1} n_{13}^{-1}$. Then $L=n_{58}^{-1} n_{67}^{-1} \Gamma n_{67} n_{58}$. Since $n_{13} n_{49} \in N\left(L_{1}\right)$ and $n_{58} n_{67} \in N\left(L_{6}\right), L$ is joined to $L_{1}$ and $L_{6}$ unitarily since $\Gamma$ is.

In $G$ we have defined the elements $h_{i}, n_{i}$ such that $n_{i} h_{i} n_{i}^{-1}=h_{i}^{-1}$ and $n_{i} h_{1} n_{i}^{-1}=h_{i} h_{i}=n_{j} h_{i} n_{j}^{-1}$ if $\{i, j\}$ is an edge. Let $h_{1}=x_{1} h_{5} x_{1}^{-1}, n_{1}=x_{1} n_{5} x_{1}^{-1}$; $h_{L}=x_{2} h_{7} x_{2}^{-1}$ and $n_{L}=x_{2} n_{7} x_{2}^{-1}$ where $x_{1}=n_{4} n_{3} n_{2} n_{4} n_{2}^{-1} n_{3}^{-1}$ and $x_{2}=$ $n_{6} n_{5} n_{\Gamma} n_{6} n_{\Gamma}^{-1} n_{5}^{-1}$. So by (2.1), we have $n_{1} \cdot h_{4} n_{\Gamma}^{-1}=h_{4} h_{\Gamma}^{-1} ; n_{\Gamma} h_{6} n_{\Gamma}^{-1}=h_{6} h_{\Gamma}$ and $n_{L} h_{6} n_{L^{-}}^{-1}=h_{6} h_{L}^{-1}$. Similarly working with the subgroup $\left\langle L_{s}, L_{6}, L_{7}, \Gamma, L_{1}, L_{3}\right\rangle$ we obtain that $n_{L} h_{1} n_{L}^{-1}=h_{1} h_{L}, n_{19} h_{1} n_{19}^{-1}=h_{1} h_{19}^{-1}$. Also by (2.1) we have the identities $h_{2} h_{3} h_{4}^{2} h_{8}=h_{\Gamma} ; h_{5} h_{6}^{2} h_{1} h_{7}=h_{L}$ and $h_{3} h_{1}^{2} h_{\Gamma} h_{L}=h_{19}$.

Now set $z_{1}=n_{L} n_{19} n_{\Gamma} n_{3} \quad\left(\right.$ resp. $h_{1} h_{L} n_{L} n_{19} n_{\Gamma} n_{3}$ ); $z_{4}=n_{\Gamma}^{-1} n_{3} n_{5} n_{2}$ (resp. $\left.h_{4} h_{5} h_{\Gamma}^{-1} n_{l}^{-1} n_{3} n_{5} n_{2}\right)$ and $z_{6}=n_{\Gamma} n_{5} n_{l}^{-1} n_{7}$ (resp. $h_{6}^{-1} h_{\Gamma}^{-1} h_{L} n_{\Gamma} n_{5} n_{L}^{-1} n_{7}$ ) when $q \equiv$ $1(4)$ (resp. $q \equiv-1(4)$ ). We compute that $z_{1}, z_{4}, z_{6}$ are commuting involutions such that $z_{1} z_{4}, z_{4} z_{6} z_{1} z_{6}$ are not conjugate to $z_{1}, z_{4}, z_{6}$ in $M_{1}, M_{2}$ and $\left\langle L_{5}, L_{6}, L_{7}, \Gamma, L_{1}, L_{3}\right\rangle$ respectively. Therefore there exist subgroup $\Gamma_{1}, \Gamma_{4}, \Gamma_{6}$ isomorphic to $S L(2, q)$ with $Z\left(\Gamma_{i}\right)=\left\langle z_{i}\right\rangle i=1,4,6$ such that $\left[\Gamma_{1}, \Gamma_{4}\right]=$ $\left[\Gamma_{4}, \Gamma_{6}\right]=\left[\Gamma_{1}, \Gamma_{6}\right]=1 ; \Gamma_{1}$ is joined to $L_{2}$ linearly; $\Gamma_{4}$ is joined to $L_{2}, L_{3}, L_{5}$ linearly and $\Gamma_{6}$ joined to $L_{5}, L_{7}$ linearly by $(2.5)$. Clearly we also have $\left[\Gamma_{1}, L_{i}\right]=1 i=2,5,7 ;\left[\Gamma_{4}, L_{7}\right]=1$ and $\left[\Gamma_{6}, L_{j}\right]=1 j=2,3,4$.

We can now apply Curtis' Theorem 1.4 to the chain of subgroups $\Gamma_{1}, L_{2}$, $L_{3}, \Gamma_{4}, L_{5}, \Gamma_{6}, L_{7}$ which generate $G$ and get that $G \cong E_{7}(q)$, the universal Chevalley group of type $E_{7}$ over $F_{0}$.

THEOREM 2.8. Let $G$ be a universal group of type $E_{\mathrm{x}}$ generated by $S U(3, q)$ 's. Then $G$ is isomorphic to the universal group of type $E_{8}$ over $F_{0}$.

Proof. Let the graph of $G$ be as follows

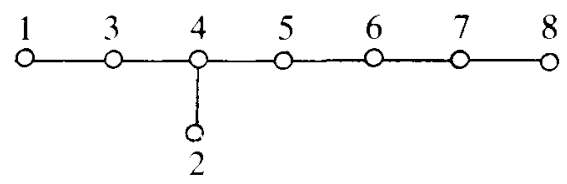

We shall use the notation in the proof of (2.7) as $\left\langle L_{i} \mid 1 \leqq i \leqq 7\right\rangle \cong E_{7}(q)$. There we have defined subgroups $L_{01}=L_{84}, L_{14}, \Gamma, L$ and $\psi=n_{17} n_{36} n_{45} n_{89}$. The 
element $\psi$ interchanges the elements of the sets $\left\{L_{3}, L_{5}\right\} ;\left\{L_{1}, L_{6}\right\}$ and $\left\{L_{19}, L_{7}\right\}$ by conjugation. Since $\psi L_{8} \psi^{-1}=L_{8}, L_{8}$ is joined to $L_{19}$ unitarily. Also $\left[L_{0}, L_{8}\right]=1$ because $L_{0} \subseteq\left\langle L_{i} \mid 1 \leqq i \leqq 6\right\rangle$.

Next we note that $Q_{1}=\left\langle L_{19}, L_{i} \mid 2 \leqq i \leqq 8\right\rangle$ is a universal group of type $D_{8}$ with $\left\langle L_{i} \mid 2 \leqq i \leqq 7\right\rangle$ as a subgroup of type $D_{6}$. In the proof of (2.7), we found that $L$ is the dual of $L$, in $Q_{1}$ and therefore by $(2.1), L$ is joined to $L_{8}$ unitarily. Let $\Gamma_{0}$ be the dual of $L_{19}$ in $Q_{1}$. We note that $\Gamma_{0} \subseteq\left\langle L_{19}, L, L_{7}, L_{8}\right\rangle$, a group of type $D_{4}$. Let $z_{1}, z_{4}, z_{6}, \Gamma_{1}, \Gamma_{4}, \Gamma_{6}$ be as defined in (2.7). Let $z_{8}=$ $h_{8} h_{19} h_{L} n_{L}^{-1} n_{19} n_{7} n_{0}$ where $n_{0}=x n_{7} x^{-1}, x=n_{8} n_{L} n_{19} n_{8} n_{19}^{-1} n_{L}^{-1}$ and $h_{0}=x h_{7} x^{-1}$. We compute that $n_{0} h_{8} n_{0}^{-1}=h_{8} h_{0}^{-1} ; n_{L} h_{8} n_{L}^{-1}=h_{8} h_{L}=n_{8} h_{L} n_{8}^{-1} ; n_{19} h_{8} n_{19}^{-1}=$ $h_{8} h_{19}=h_{8} h_{19} n_{8}^{-1}$. Together with the relations found in (2.7), we compute that $z_{1}, z_{8}, z_{6}$ are commuting involutions such that $z_{1} z_{8}, z_{8} z_{6}$ are not conjugate to $z_{1}, z_{8}$ in $\left\langle L_{3}, L_{1}, \Gamma, L, L_{8}, L_{7}\right\rangle$ and $\left\langle L_{5}, L_{6}, \Gamma, L_{7}, L_{8}, L_{19}\right\rangle$ respectively. It follows by (2.5). $\left[\Gamma_{1}, \Gamma_{8}\right]=\left[\Gamma_{8}, \Gamma_{6}\right]=1$ where $\Gamma_{8}$ is the unique subgroup isomorphic to $S L(2, q)$ in $\left\langle L_{19}, \Gamma_{0}, L_{7}, L_{8}, L\right\rangle$ and also $\Gamma_{8}$ is joined to $L_{7}$ linearly. From the proof of (2.7), $\left\langle L, L_{19}\right\rangle$ centralizes $\left\langle L_{i} \mid 2 \leqq i \leqq 5\right\rangle$; hence $\left[\left\langle L, L_{19}, L_{8}, L_{7}\right\rangle\right.$, $\left.\left\langle L_{2}, L_{3}, L_{4}, L_{5}\right\rangle\right]=1$ and therefore $\left[\Gamma_{8}, L_{i}\right]=1=\left[\Gamma_{8}, \Gamma_{4}\right] i=2,3,4$ because $\Gamma_{4} \subseteq$ $\left\langle L_{2}, L_{3}, L_{4}, L_{5}\right\rangle$ and $\Gamma_{8} \subseteq\left\langle L, L_{19}, L_{8}, L_{7}\right\rangle$. Finally we compute that the chain of subgroups $\Gamma_{1}, L_{2}, L_{3}, \Gamma_{4}, L_{5}, \Gamma_{6}, L_{7}, \Gamma_{8}$ generates $G$ and by Curtis' Theorem 1.4 [Curtis, 1965], $G \cong E_{8}(q)$, the universal Chevalley group of type $E_{8}$ over $F_{0}$. This completes the proof.

\section{References}

Charles W. Curtis (1965), 'Central extensions of groups of Lie type', J. reine angew. Math. 220, 174-185.

Jean Dieudonné (1955), La Géometrie des Groupes Classiques (Ergebnisse der Mathematik und ihrer Grenzgebiete, 5. Springer-Verlag, Berlin, Göttingen, Heidelberg, 1955).

Robert L. Griess, Jr. (1972), 'Schur multipliers of the known finite simple groups', Bull. Amer. Math. Soc. 78, 68-71.

Nagayoshi Iwahari (1970), 'Centralizers of involutions in finite Chevalley groups', Seminar on Algebraic Groups and Related Finite Groups, F1-F29 (Lecture Notes in Mathematics, 131. Sprinver-Verlag, Berlin, Heidelberg, New York, 1970).

Kok-Wee Phan (1977), 'On groups generated by three-dimensional special unitary groups', $J$. Austral. Math. Soc. 23 (Series A), 67-77.

W. J. Wong (1974), 'Generators and relations for classical groups', J. Algebra 32, 529-553.

Department of Mathematics,

University of Notre Dame,

Notre Dame, U.S.A. 